\title{
Atom-laser dynamics
}

\author{
Nicholas Robins, ${ }^{1}$ Craig Savage, ${ }^{1}$ and Elena A. Ostrovskaya ${ }^{2}$ \\ ${ }^{1}$ Department of Physics and Theoretical Physics, The Australian National University, Canberra ACT 0200, Australia \\ ${ }^{2}$ Nonlinear Physics Group, The Australian National University, Canberra ACT 0200, Australia
}

(Received 2 January 2001; published 13 September 2001)

\begin{abstract}
An ideal atom laser would produce an atomic beam with highly stable flux and energy. In practice, the stability is likely to be limited by technical noise and nonlinear dynamical effects. We investigate the dynamics of an atom laser using a comprehensive one-dimensional, mean-field numerical model. We fully model the output beam and experimentally important physics such as three-body recombination. We find that at highpump rates, the latter plays a role in suppressing the high-frequency dynamics, which would otherwise limit the stability of the output beam.
\end{abstract}

DOI: 10.1103/PhysRevA.64.043605

Optical lasers have had an enormous impact on science and technology, due to the intensity and coherence of the light they produce. We present a theoretical study of the output beam of the analogous matter wave device, known as the "atom laser." These have been demonstrated in a number of laboratories, although so far, only in an unpumped mode [1-6].

Theoretical studies have highlighted both the similarities and the differences between optical and atom lasers [7-12]. The differences arise because atoms are more complex than photons: they have mass, giving a different free space dispersion relation, and they interact with each other, producing strong nonlinearities. The latter generates complex dynamics, potentially complicating certain experimental measurements, of quantum noise for example. Nevertheless, the output beam dynamics might be useful, in itself, as a probe of the excitations of the trapped condensate $[13,14]$.

Fundamentally, it is the coherence properties, such as linewidth and intensity correlations, that best capture the unique physics of lasers $[15,16]$. Coherence is also important for many practical applications, such as interferometry. A classical nonlinear model of the laser as a noise-driven van der Pol oscillator shows that the noise power, in both the phase and the amplitude, decreases in inverse proportion to the laser power [17].

Schawlow and Townes showed that optical lasers' firstorder coherence, or linewidth, is ultimately limited by spontaneous emission, and also decreases in inverse proportion to the laser power [18]. Analogous results have been derived for atom lasers [19]. In practice, however, the SchawlowTownes limit is not achieved, because the linewidth is limited by technical noise and by dynamical effects rather than by quantum noise. For example relaxation oscillations, due to the nonlinear interaction between the inversion and the light, are the primary determinant of the low-frequency noise spectrum in many optical lasers [20].

In this paper, we focus on one aspect of the nonlinear dynamics of atom lasers: the frequency spectrum of the atom-laser output beam density. In particular, we investigate how the spectrum depends on the pumping rate. This spectrum describes the atom-laser nonlinear dynamics. An experimentally measured spectrum would also include genuine "noise" due to physical processes that we do not model, such as fluctuations in the trapping potential. This investiga-
PACS number(s): 03.75.Be

tion is motivated by the inverse relation between noise power and pumping rate in the optical laser. We choose to focus on the density because it can be measured experimentally using sensitive optical techniques such as phase-modulation spectroscopy [21].

A conclusion of our paper is that three-body recombination plays a major role in the high-frequency dynamics. As the pumping rate is increased, the spectral peaks weaken and move to higher frequencies. This knowledge will facilitate atom-laser applications, and measurements of their quantum noise.

Our atom laser model is schematically illustrated in Fig. 1. It consists of a dilute gas Bose-Einstein condensate trapped by a highly anisotropic, effectively one-dimensional, potential. This is pumped from a reservoir of noncondensed trapped atoms. The output atom-laser beam is generated by a Raman transition to an untrapped atomic state. Our model builds on that of Kneer et al. [11] by adding three-body recombination and a propagating output beam. In the following, we will refer to the trapped Bose-Einstein condensate as the "condensate," and to the output beam as the "beam." We make the mean-field approximation in which the condensate and beam are described by the classical fields $\Psi_{a}(x, t)$ and $\Psi_{b}(x, t)$, respectively, which obey Gross-Pitaevskii type equations [22]. Our model is more complete than previous

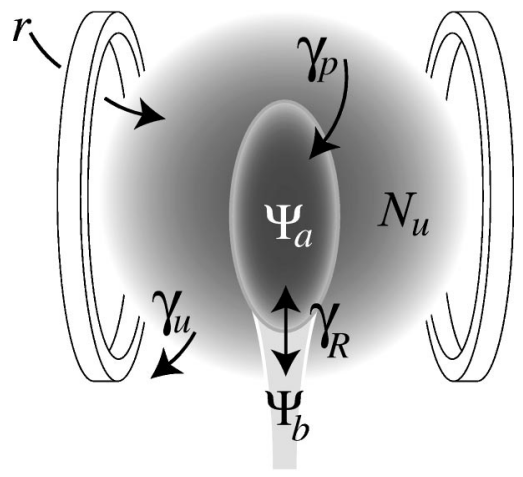

FIG. 1. Schematic representation of our atom-laser model. The rings represent coils producing the trapping potential. The symbols are defined in the text. Atoms are injected into the noncondensed fraction. They can then either be lost or pump the condensate. They are coupled out of the condensate by a Raman transition and then fall in the gravitational field. 
mean-field treatments $[11,23-26]$ since we include pumping, output coupling, three-body recombination, and explicitly model the dynamics of the pump reservoir, the condensate, and the beam. Although the extension to three dimensions is straightforward, we do not report it here. To describe the pumping, we use a phenomenological model, which mimics the pumping of an optical laser [11]. It only depends on the total number of atoms in the uncondensed component $N_{u}(t)$, not on its spatial structure. The condensate atoms are coupled, by a reversible Raman transition [2,23,27], to an untrapped electronic state forming the atom-laser beam. The Raman transition imparts a momentum kick $\hbar k$ to the outcoupled atoms. The beam evolves under the influences of gravity and atom-atom interactions, which are dominated by those due to the condensate. We have only considered the particular case for which the Raman coupling is tuned to transfer atoms starting from the center of the harmonic trap [23]. Hence, the output beam overlaps with the lower half of the condensate. Experimentally, a three-body recombination is well described as a loss process occurring at a rate proportional to the cube of the local density [28]. We follow Kagan et al. [29] in incorporating a three-body recombination into the atom laser Gross-Pitaevskii equations.

Our model is defined by the following dimensionless equations:

$$
\begin{gathered}
\frac{d N_{u}}{d t}=r-\gamma_{u} N_{u}-\gamma_{p} N_{u} N_{a}, \\
i \frac{\partial \Psi_{a}}{\partial t}=-\frac{1}{2} \frac{\partial^{2} \Psi_{a}}{\partial x^{2}}+\frac{1}{2} x^{2} \Psi_{a}+U_{a} \Psi_{a}\left|\Psi_{a}\right|^{2}+U_{a b} \Psi_{a}\left|\Psi_{b}\right|^{2} \\
-i \gamma_{r} \Psi_{a}\left(\left|\Psi_{a}\right|^{4}+\left|\Psi_{b}\right|^{4}\right)+\gamma_{R} e^{i k x} \Psi_{b}+\frac{i}{2} \gamma_{p} N_{u} \Psi_{a}, \\
i \frac{\partial \Psi_{b}}{\partial t}=-\frac{1}{2} \frac{\partial^{2} \Psi_{b}}{\partial x^{2}}+G x \Psi_{b}+U_{b} \Psi_{b}\left|\Psi_{b}\right|^{2}+U_{a b} \Psi_{b}\left|\Psi_{a}\right|^{2} \\
-i \gamma_{r} \Psi_{b}\left(\left|\Psi_{a}\right|^{4}+\left|\Psi_{b}\right|^{4}\right)+\gamma_{R} e^{-i k x} \Psi_{a} .
\end{gathered}
$$

The model is made dimensionless using the characteristic trap length $l=(\hbar / \omega m)^{1 / 2}$ and angular frequency $\omega$, with $m$ the atomic mass. Hence, time $t$, position $x$, and the fields are measured in units of $\omega^{-1}, l$ and $l^{-1 / 2}$, respectively. Experimentally reasonable values of the parameters are a trap frequency $\omega \approx 125 \mathrm{~Hz}$ [30], and the atomic mass of sodium $m=3.8 \times 10^{-26} \mathrm{~kg}$. These give a time scale of $\omega^{-1}=8 \mathrm{~ms}$, and a length scale of $l=4.7 \mu \mathrm{m}$.

Atoms are injected into the uncondensed fraction at the rate $r$, are lost at the rate $\gamma_{u} N_{u}$, and pump the condensate at the rate $\gamma_{p} N_{u} N_{a}$, where $N_{a}=\int_{-\infty}^{\infty}\left|\Psi_{a}\right|^{2} d x$ is the total condensate population. $U_{a}$ and $U_{b}$ are the intra- and $U_{a b}$ the inter- species two-body interaction coefficients. The dimensionally correct coefficients can be written as $U_{a, b, a b}$ $=4 \pi a_{s} /(\hbar / \omega m)^{1 / 2}$ with $a_{s}$ the appropriate $s$-wave scattering lengths [23]. We have previously found that the three-body recombination, with coefficient $\gamma_{r}$, is necessary for the sys-

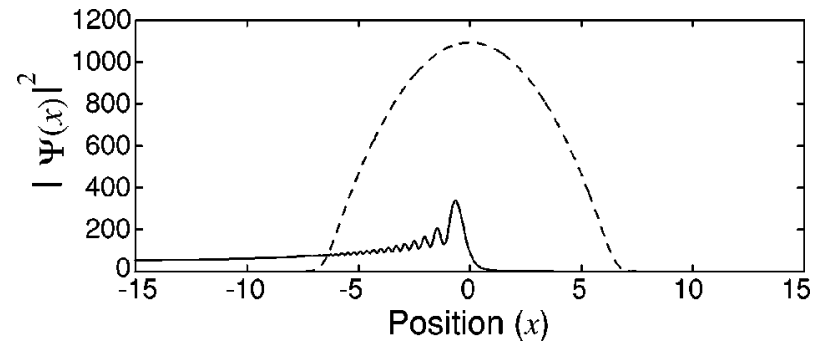

FIG. 2. Densities of the condensate (dashed) and beam (solid) versus position. All quantities are dimensionless. The plotted beam density is 100 times the true value. These results are a numerical solution of Eqs. (1) at $t=200$ after starting from a seed condensate. Parameters are $r=1600, U_{a}=U_{b}=0.02, U_{a b}=0.01, G=12, \gamma_{R}$ $=0.5, \gamma_{u}=\gamma_{p}=0.1, \gamma_{r}=10^{-7}$, and $k=5$.

tem to reach a quasi-stationary state on a timescale comparable to experimental condensate lifetimes [31]. The threebody recombination rate $\gamma_{r}=10^{-7}$ is chosen to produce $N_{a}$ $\approx 3500-9500$ condensed atoms at a steady state, as the pump rate varies from $r=200$ to 1600 . For simplicity we assume that the inter- and intra-species three-body recombination rates are the same. $G$ is the scaled gravitational acceleration [32]. Gravity has not been explicitly included for the trapped atoms, since it is equivalent to a spatial shift of the trap potential. The Raman coefficient $\gamma_{R}$ is a function of the Raman laser amplitude and detuning [27].

Our numerical method is a split-step Fourier method [33] with a fourth-order Runge-Kutta in place of the usual nonlinear step. We have absorbing boundaries at the ends of the spatial grids to prevent reflections.

Typical results for quasistationary-state spatial profiles of the condensate and beam densities are shown in Fig. 2. For our parameters, the condensate shape is well described in the Thomas-Fermi approximation. Note that the plotted output beam density has been scaled up by a factor of 100 . The flux in the beam is about 90 atoms per unit dimensionless time, or about $1.1 \times 10^{4}$ atoms per second with $\omega=125 \mathrm{~Hz}$. The prominent spatial oscillations of the output beam, occurring within the extent of the condensate, have been observed in previous work $[24,34]$ and are related to the oscillatory Airy type eigenfunctions of particles in the linear gravitational potential.

In Fig. 3, we show the output beam density $\left|\Psi_{b}\left(x_{0}, t\right)\right|^{2}$ as a function of time at a fixed position far from the trapped condensate. The time span has been chosen to be longer than that of the transient dynamics associated with the growth of the condensate from a seed, and short enough to be experimentally accessible. Quasistationary, nearly periodic dynamics develop, becoming approximately harmonic for large pump rates $r$. We have verified that the quasistationary dynamics is independent of the particular form of the seed used to initiate the condensate growth. The fundamental oscillation of the beam, at higher pump rates, arises from the wellknown Kohn mode [22], corresponding to a rigid "sloshing" back and forth of the condensate at the trap frequency.

Figure 4 shows the frequency spectra of the density time series in Fig. 3, and is the central result of this paper. The spectra are those of the final, quasisteady, parts of the time 


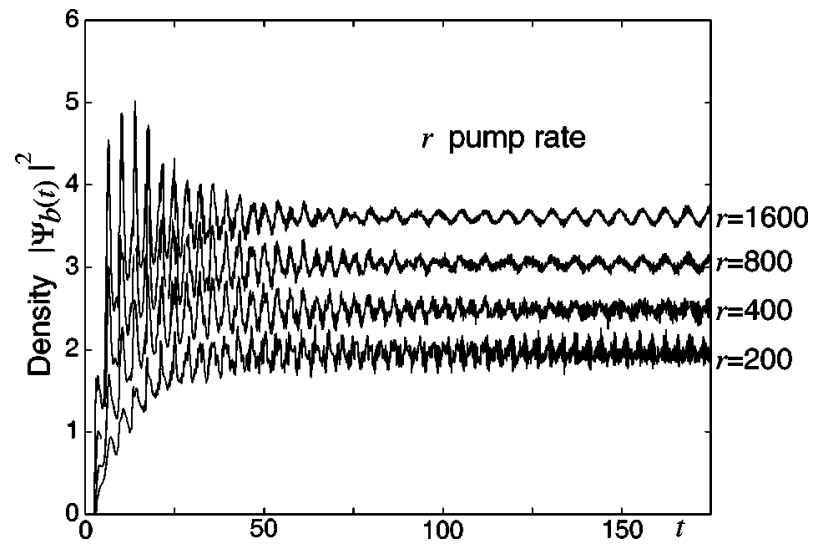

FIG. 3. The output beam density, $\left|\Psi_{b}\left(x_{0}, t\right)\right|^{2}$, as a function of time at position, $x_{0}=-45$, for different pump rates: from bottom to top $r=200,400,800$, and 1600. All quantities are dimensionless. Other parameters are as for Fig. 2.

series. The frequencies are well approximated by the eigenfrequencies of an uncoupled one-dimensional (1D) condensate. Kneer et al. [11] found these to be $\Omega=\omega \sqrt{n(n+1) / 2}$ where $n=1,2,3, \ldots$, with the corresponding spatial modes given by the Legendre polynomials $P_{n}(x)$. The Kohn sloshing mode at $\omega$ is $n=1$, and $n=2$ is the breathing mode at $\sqrt{3} \omega$. In this mode, the width of the condensate oscillates, with a corresponding density oscillation.

Figure 4 shows that as the pumping rate $r$ increases, the slow beam oscillations change from the frequency of the condensate breathing mode to that of the sloshing mode. This is because the condensate itself changes from breathing to sloshing at higher pumping rates. As Kneer et al. [11] noted, the sloshing mode is spatially asymmetric, and hence, must be excited by a spatially asymmetric perturbation, such as the output beam. This is consistent with the observed increase of the sloshing-mode power with the density of the output beam, that is with pumping rate. On the other hand, mode damping due to three-body recombination, increases rapidly with density. We have verified that in a simple model of the Kneer et al. [11] form, the breathing mode decays with pumping rate.

Although the frequencies in the beam are primarily determined by the condensate, there is a complex relationship between the power spectra of the condensate and of the beam. In particular, the beam spectrum reflects the spatial dependence of the condensate spectrum. Consider, for example, the condensate Kohn sloshing mode. The magnitude of the local-density change varies with the spatial derivative of the density profile. It is maximum where the condensate has greatest slope, and zero at the center where the slope is zero. That the Kohn-mode frequency is prominent in the

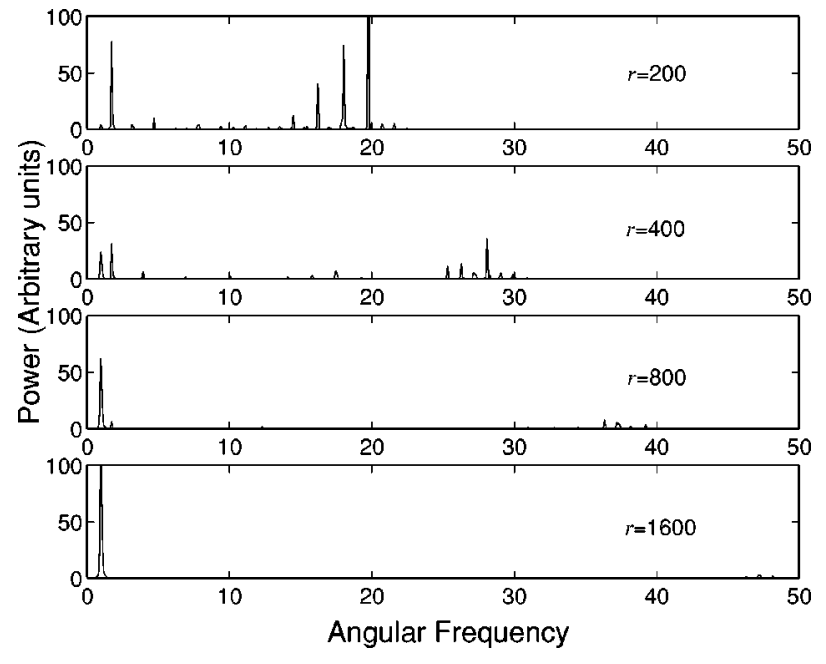

FIG. 4. Frequency spectra of the time-dependent density of the fields in Fig. 3. All quantities are dimensionless. The spectra are those of the time series from $t=112.5$ to $t=175$. The pump rates from top to bottom are $r=200,400,800$, and 1600 . The normalization has been chosen so that the largest peak height (that near 20 frequency units for $r=200$ ) is 100 units. The peaks just below 50 frequency units, for $r=1600$, are almost invisible on this scale.

power spectrum of the output beam, indicates that the coupling between the condensate and the beam is not confined to the center of the condensate. Similar observations apply for the high-frequency components of the power spectrum, the envelopes of which differ in detail between the condensate and the beam.

As the pumping rate increases, Fig. 4 shows that the frequency of the high-frequency group increases and its power decreases rather dramatically. A decrease of spectral power with pumping rate, due to gain saturation, is characteristic of optical lasers. Since three-body recombination contributes to gain saturation in atom lasers, we have investigated its role in a simplified pumped atom-laser model similar to that of Kneer et al. [11]. That is, with a spatially uniform phenomenological loss rather than an explicitly modeled output beam. Without a three-body recombination, the condensate density power spectrum simply rolls off at high frequencies. Adding a three-body recombination produces a highfrequency spectral group, such as seen in Fig. 4. Hence, it is an important factor in the dynamics of the system.

In conclusion, we have explored some of the rich and complex behavior of the atom laser with a model incorporating important experimentally relevant physics, such as the three-body recombination. The latter was found to strongly influence the spectrum of the atom-laser output beam. This paper is a step towards the future goal of understanding and measuring the quantum noise properties of the atom laser.
[1] M.-O. Mewes, M.R. Andrews, D.M. Kurn, D.S. Durfee, C.G. Townsend, and W. Ketterle, Phys. Rev. Lett. 78, 582 (1997).

[2] E.W. Hagley, L. Deng, M. Kozuma, J. Wen, K. Helmerson, S.L. Rolston, and W.D. Phillips, Science 283, 1706 (1999).
[3] I. Bloch, T.W. Hansch, and T. Esslinger, Phys. Rev. Lett. 82, 3008 (1999).

[4] I. Bloch, T.W. Hansch, and T. Esslinger, Nature (London) 403, 166 (2000). 
[5] B.P. Anderson and M.A. Kasevich, Science 282, 1686 (1998).

[6] J.L. Martin et al., J. Phys. B 32, 3065 (1999).

[7] H. Wiseman and M. Collett, Phys. Rev. A 202, 246 (1995).

[8] H. Wiseman, A. Martins, and D. Walls, Quantum Semiclassic. Opt. 8, 737 (1996).

[9] M. Holland, K. Burnett, C. Gardiner, J.I. Cirac, and P. Zoller, Phys. Rev. A 54, R1757 (1996).

[10] J.J. Hope, G.M. Moy, M.J. Collett, and C.M. Savage, Phys. Rev. A 61, 023603 (2000).

[11] B. Kneer, T. Wong, K. Vogel, W.P. Shleich, and D.F. Walls, Phys. Rev. A 58, 4841 (1998).

[12] M. Naraschewski, A. Schenzle, and H. Wallis, Phys. Rev. A 56, 603 (1997).

[13] Y. Japha, S. Choi, K. Burnett, and Y. Band, Phys. Rev. Lett. 82, 1079 (1999).

[14] S. Choi, Y. Japha, and K. Burnett, Phys. Rev. A 61, 063606 (2000).

[15] L. Mandel and E. Wolf, Optical Coherence and Quantum Optics. (Cambridge University Press, Cambridge, MA, 1995).

[16] R. J. Ballagh and C. M. Savage, Bose-Einstein Condensation: From Atomic Physics to Quantum Fluids, Proceedings of the 13th Physics Summer School, edited by C. M. Savage and M. Das (World Scientific, Singapore, 2000).

[17] J.A. Armstrong and A.W. Smith, Prog. Opt. 6, 211 (1967).

[18] A.L. Schawlow and C.H. Townes, Phys. Rev. 112, 1940 (1958).

[19] R. Graham, Phys. Rev. Lett. 81, 5262 (1998).

[20] A. Yariv, Quantum Electronics (Wiley, New York, 1989).

[21] J.E. Lye, B.D. Cuthbertson, H.-A. Bachor, and J.D. Close, J. Opt. B: Quantum Semiclassical Opt. 1, 402 (1999).

[22] F. Dalfovo, S. Giorgini, L.P. Pitaevskii, and S. Stringari, Rev. Mod. Phys. 71, 463 (1999).
[23] M. Edwards, D.A. Griggs, P.L. Holman, C.W. Clark, S.L. Rolston, and W.D. Phillips, J. Phys. B 32, 2935 (1999).

[24] J. Schneider and A. Schenzle, Appl. Phys. B: Lasers Opt. 69, 353 (1999).

[25] R.J. Ballagh, K. Burnett, and T.F. Scott, Phys. Rev. Lett. 78, 1607 (1997).

[26] Y.B. Band, P.S. Julienne, and M. Trippenbach, Phys. Rev. A 59, 3823 (1999).

[27] G.M. Moy, J.J. Hope, and C.M. Savage, Phys. Rev. A 55, 3631 (1997).

[28] E.A. Burt, R.W. Ghrist, C.J. Myatt, M.J. Holland, E.A. Cornell, and C.E. Wieman, Phys. Rev. Lett. 79, 337 (1997).

[29] Yu. Kagan, A.E. Muryshev, and G.V. Shlyapnikov, Phys. Rev. Lett. 81, 933 (1998).

[30] M.-O. Mewes, M.R. Andrews, N.J. van Druten, D.M. Kurn, D.S. Durfee, and W. Ketterle, Phys. Rev. Lett. 77, 416 (1996).

[31] N. Robins, C. Savage, and E. Ostrovskaya, in Walls Memorial Volume, edited by H. J. Carmichael et al. (Springer, New York, 2000).

[32] For reasons of computational efficiency, we found it convenient to use $G=12$. This is because higher values produce faster atoms with shorter de Broglie wavelengths, requiring smaller spatial grid sizes, and hence, bigger computations. For the preceding parameters, $g=9.8 \mathrm{~ms}^{-2}$ corresponds to $G$ $=132$. Physically, low- $G$ values might correspond to the atomlaser beam propagating in a tilted wave guide. Alternatively, a trap frequency about five times higher $(\omega \approx 625 \mathrm{~Hz})$ would make $G=12$ correspond to $g=9.8 \mathrm{~ms}^{-2}$.

[33] T.R. Taha and M.J. Ablowitz, J. Comp. Physiol. 55, 203 (1984).

[34] J. Schneider and A. Schenzle, Phys. Rev. A 61, 053611 (2000). 